\title{
A stochastic control model of economic growth with environmental disaster prevention ${ }^{\text {tr }}$
}

\author{
Alain Haurie $^{\mathrm{a}, *}$, Francesco Moresino $^{\mathrm{b}}$ \\ ${ }^{a}$ HEC-LOGILAB, University of Geneva, Switzerland \\ ${ }^{\mathrm{b}}$ Ecole Polytechnique Fédérale de Lausanne (EPFL) and HEC-LOGILAB, University of Geneva, Switzerland
}

Received 16 December 2004; received in revised form 19 August 2005; accepted 25 October 2005

Available online 20 January 2006

\begin{abstract}
This paper proposes a capital accumulation model with a random stopping time corresponding to the occurrence of an environmental catastrophe. Depending on the preventive capital stock accumulated at the time of the catastrophe, the damage cost associated with the catastrophe varies. The long-term behavior of the optimal accumulation path is analyzed using turnpike theory. The case where the catastrophe process is uncontrolled is distinguished from the case where there is an anthropogenic effect on the probability of an occurrence. Intergenerational equity issues are discussed. Numerical experiments with an adaptation of the integrated assessment model DICE94 are proposed to explore the model responses.
\end{abstract}

(C) 2005 Elsevier Ltd. All rights reserved.

Keywords: Stochastic control; Stochastic jump processes; Preventive control; Optimal economic growth; Global climate risk

\section{Introduction}

An eventual climate change may trigger in a possibly distant future large scale catastrophic events like a 5-6 m sea level rise, due to a collapse of the West-Antarctic Ice Sheet. The aim of this paper is to explore the relevance to the analysis of economic decisions in this context of an economic growth model with random stopping time, representing the occurrence of a catastrophic event. The economic growth model is akin to Ramsey's (Ramsey, 1928) capital accumulation model as revisited by Arrow and Kurz (1970), Cass (1965), Cass and Shell (1976) and many others. The new feature is the inclusion of a random stopping time at which a significant catastrophic event occurs. The model takes then the form of a control problem with a random stopping time as treated in Boukas, Haurie, and Michel (1990). One also assumes that the economic good can be invested in two different physical capital stocks, one being

\footnotetext{
is This paper was not presented at any IFAC meeting. This paper was recommended for publication in revised form by Associate Editor Qing Zhang under the direction of Editor Suresh Sethi.

* Corresponding author. HEC Uni-Mail, 40 Blvd. du Pont-d'Arve, 1211 Geneva 4, Switzerland. Tel.: +41 22705 8132; fax: +41 227058104.

E-mail address: alain.haurie@hec.unige.ch (A. Haurie).
}

the general productive physical capital and the other being an equipment that will alleviate the consequences (social cost) of the catastrophe when it occurs. We develop two variants of the economic growth model in the presence of catastrophic events that cover the cases where (i) the stopping time of catastrophe occurrence is uncontrolled, (ii) the stopping time probability measure is influenced by the economic activity, respectively. Assuming that the concentration of GHGs ${ }^{1}$ in the atmosphere has an influence on the catastrophe risk one can view the problem as an instance of the derivation of a climate impact response function (Tóth, Cramer, \& Hizsnyik, 2000). All these models draw from the theory of asymptotic control exposed in Carlson, Haurie, and Leizarowitz (1994) and exploit the relevance of infinite horizon control models to the study of sustainable development (Haurie, 2003).

An interesting feature of these models is the very long time horizon allied with a low intensity of the catastrophe jump process. We shall discuss the way intergenerational equity can be represented in this class of models and how this influences the long-term economic behavior which is represented by "turnpikes", which are attractors of the trajectories. The turnpike property for piecewise deterministic systems has been first studied in Fleming, Sethi, and Soner (1987) and extended in

\footnotetext{
${ }^{1}$ Greenhouse gases.
} 
Haurie and Van Delft (1995) in the context of manufacturing systems. Another feature of this class of models is that a numerical analysis is essential for exploring the possible outcomes, in contrast with classical economic growth theory which may rely essentially on analytic results and their interpretation. We thus develop in detail the implementation of numerical methods for this stochastic control problem as an example of a computational economics approach.

The paper is organized as follows: in Section 2 we consider the case of an economy facing a stochastic catastrophic event and which can invest in protective equipment or facilities; we distinguish the two sub-classes corresponding to the uncontrolled catastrophe process (similar to a meteoric collision or a big earthquake) and to the anthropogenically influenced catastrophe process (typically, the threshold events triggered by climate warming), respectively; in both cases we provide the necessary optimality conditions and we characterize the possible turnpikes. We also discuss the intergenerational equity issue and the choice of an appropriate pure time preference rate in the welfare criterion (defined as the infinite horizon discounted sum of consumption utility). In Section 3 we detail the method for computing numerical solutions of these models and we discuss the results obtained for both classes of models. In conclusion, we envision some further developments of this avenue of research.

\section{The model}

In this section we propose two versions of an economic growth model with random catastrophic event. The first version deals with an uncontrolled catastrophe process, whereas the second version includes an anthropogenic influence on the catastrophe elementary probability of occurrence.

\subsection{The case of an uncontrolled catastrophe}

Consider an economy characterized by a malleable good that is produced with labor $L$, supposed to be fixed, and a physical capital $K_{1}$, according to a production function $Y=f^{i}\left(K_{1}\right)$ where $Y$ is the output and $f^{i}(\cdot)$ is a concave function ${ }^{2}$ satisfying the classical Inada conditions, $i=0,1$ (see Arrow \& Kurz, 1970). The index $i$ represents two different modes of the economy (before and after the catastrophe, respectively) that will be described shortly. There is a second physical capital stock, denoted $K_{2}$ whose role is to mitigate the consequences (damages) of a possible disaster, like the breaking of the antarctic ice shield and the possible sudden rise of the sea level, or the collision of the planet with a large asteroid. ${ }^{3}$ The output $Y$ can be used for consumption $C$ or investment $I$.

\footnotetext{
${ }^{2}$ For the sake of simplicity, we have assumed a constant population and no exogenous technological progress.

3 The asteroid collision is certainly an uncontrolled event, whereas the ice shield breaking is probably influenced by anthropogenic climate change which is therefore a controlled process. This case is considered later on in the paper.
}

\subsubsection{Economic and catastrophe dynamics}

The economy dynamics is characterized by the productive capital accumulation equation

$\dot{K}_{1}(t)=I_{1}(t)-\delta_{1} K_{1}(t)$,

where $\delta_{1}$ is the capital depreciation rate, and the preventive capital accumulation

$\dot{K}_{2}(t)=I_{2}(t)-\delta_{2} K_{2}(t)$

with its own depreciation rate $\delta_{2}$. The state of the economy at time $t$ is described by the hybrid variable $s(t)=(\mathbf{K}(t), \zeta(t))$ where $\mathbf{K}(t)=\left(K_{1}(t), K_{2}(t)\right)$ describes the accumulated stocks of both types of capital and $\zeta(t) \in\{0,1\}$ is a discrete variable indicating the mode of the economy: $\zeta=0$ is the current situation, whereas $\zeta=1$ corresponds to the situation where a catastrophic event has occurred, like the breaking of the antarctic ice shield or the "nuclear winter" that would follow a collision with an asteroid. We assume that the occurrence of the catastrophe is well described as a Poisson process with intensity $\lambda$. That is equivalent to say that the process $\{\zeta(t): t \geqslant 0\}$ is a Markov chain with constant transition rate

$\lambda=\lim _{\mathrm{d} t \rightarrow 0} \frac{\mathrm{P}[\zeta(t+\mathrm{d} t)=1 \mid \zeta(t)=0]}{\mathrm{d} t}$

and where we assume that the mode $\zeta=1$ is a trapping state, which means that there is no possibility to return to mode $\zeta=0$, i.e.

$0=\lim _{\mathrm{d} t \rightarrow 0} \frac{\mathrm{P}[\zeta(t+\mathrm{d} t)=0 \mid \zeta(t)=1]}{\mathrm{d} t}$.

Let $T$ denote the random (stopping) time at which the catastrophe occurs. We assume that there will be a large social cost, due to the loss of lives or morbidity increase, as well as a loss of productive capital. We assume that the stock $K_{2}(T)$ of preventive capital available at the time of the catastrophe has an influence on the social cost, represented by the function $\Psi\left(K_{2}\right)$; the higher the capital stock the lower this cost. Another cost is introduced in the model; it is defined as an impulse change in the stock of productive capital $K_{1}(T)$. We denote by $\Phi\left(K_{1}\left(T^{-}\right), K_{2}\left(T^{-}\right)\right)$the magnitude of the capital loss. ${ }^{4}$ This loss depends on the size of the capital stock $K_{1}$ as the more capital intensive is the society, the more exposed is this capital to a catastrophe impact. It also depends on the stock of preventive capital available which is assumed to provide a protection at the time of the impact. We represent the impulse jump of $K_{1}$ at the catastrophe time as follows:

$K_{1}\left(T^{+}\right)=K_{1}\left(T^{-}\right)-\Phi\left(K_{1}\left(T^{-}\right), K_{2}\left(T^{-}\right)\right)$.

Notice that we do not represent the impact on $K_{2}$ because this capital will have no further use after the catastrophe.

\footnotetext{
${ }^{4} T^{-}$and $T^{+}$denote the time right before and right after the catastrophe occurrence, respectively.
} 


\subsubsection{Optimal capital accumulation path}

The welfare function of the representative single agent is defined as

$J=E\left[\int_{0}^{\infty} \mathrm{e}^{-\rho t} U(C(t)) \mathrm{d} t-\mathrm{e}^{-\rho T} \Psi\left(K_{2}(T)\right)\right]$,

where $U(\cdot)$ is a non-decreasing concave utility function of consumption and $\rho$ is the pure time preference discount rate. The expression (6) is hiding the fact that there is a change in the dynamics accompanied by a state jump for $K_{1}(\cdot)$ that occur at the stopping time $T$. It is because this event is triggered by a stochastic process that we have used the expected value operator in (6). The welfare criterion can thus be rewritten as

$$
\begin{aligned}
J= & E\left[\int_{0}^{T} \mathrm{e}^{-\rho t} U(C(t)) \mathrm{d} t\right. \\
& \left.+\mathrm{e}^{-\rho T} \int_{T}^{\infty} \mathrm{e}^{-\rho(t-T)} U(C(t)) \mathrm{d} t-\Psi\left(K_{2}(T)\right)\right] .
\end{aligned}
$$

So we separate the whole time interval $[0, \infty)$ in two intervals, namely $\mathscr{I}_{0}=[0, T)$ and $\mathscr{I}_{1}=[T, \infty)$. On $\mathscr{I}_{0}$ the economy prepares for the catastrophe, whereas on $\mathscr{I}_{1}$ it recovers. Using a dynamic programming argument, we introduce a value function defined by the solution of the optimal economic growth problem on $\mathscr{I}_{1}$. Due to the stationarity of the economy description we may change the time set by translation and take $T=0$ for that problem and write

$V^{1}\left(K_{1}^{1}\right)=\max \left[\int_{0}^{\infty} \mathrm{e}^{-\rho t} U(C(t)) \mathrm{d} t\right]$

s.t.

$K_{1}\left(0^{+}\right)=K_{1}^{1}$,

$\dot{K}_{1}(t)=I_{1}(t)-\delta_{1} K_{1}(t)$,

$Y(t)=f^{1}\left(K_{1}(t)\right)$,

$C(t)=Y(t)-I_{1}(t)$

The function $V^{1}\left(K_{1}^{1}\right)$ represents the welfare prospect of the economy right after the occurrence of the catastrophe when the productive capital left is $K_{1}^{1}$. Now, conditioning on the time $T$ of the catastrophe we can define the two-stage economic growth problem as follows:

$$
\begin{aligned}
V^{0}\left(\mathbf{K}^{0}\right)= & \max E\left[\int_{0}^{T} \mathrm{e}^{-\rho t} U(C(t)) \mathrm{d} t\right. \\
& +\mathrm{e}^{-\rho T}\left(V ^ { 1 } \left(K_{1}\left(T^{-}\right)-\Phi\left(K_{1}\left(T^{-}\right), K_{2}\left(T^{-}\right)\right)\right.\right. \\
& \left.\left.\left.-\Psi\left(K_{2}(T)\right)\right)\right)\right]
\end{aligned}
$$

s.t.

$$
\begin{aligned}
& K_{j}(0)=K_{j}^{0}, \quad j=1,2, \\
& \dot{K}_{j}(t)=I_{j}(t)-\delta_{j} K_{j}(t), \quad j=1,2, \\
& Y(t)=f^{0}\left(K_{1}(t)\right),
\end{aligned}
$$

$C(t)=Y(t)-I_{1}(t)-I_{2}(t)$,

$\lambda=\lim _{\mathrm{d} t \rightarrow 0} \frac{\mathrm{P}[T \in(t, t+\mathrm{d} t) \mid T \geqslant t]}{\mathrm{d} t}$.

It will be convenient to introduce the function

$W(\mathbf{K})=V^{1}\left(K_{1}-\Phi\left(K_{1}, K_{2}\right)\right)-\Psi\left(K_{2}\right)$,

which represents the net welfare value of the economy right after the time of occurrence of the catastrophe. It is possible to rewrite the stochastic control problem defined in Eqs. (13)-(18) as a deterministic infinite horizon control problem (see, for example, Chapter 10 in Sethi, 1997). In this deterministic control problem the criterion is given by

$\int_{0}^{\infty} \mathrm{e}^{-(\rho+\lambda) t}\left\{U(C(t))+\lambda W\left(K_{1}(t), K_{2}(t)\right)\right\} \mathrm{d} t$

and the dynamic constraints are (14)-(17).

\subsubsection{Interpretation of optimality conditions}

The model developed above shows that the optimal allocation of resources to catastrophe risk prevention can be reduced to an optimal economic growth problem with welfare depending explicitly on the capital stocks. Indeed the representative agent "consumes" the protection offered by the stock of capital $K_{2}(t)$ or the exposure due to the stock of capital $K_{1}(t)$. This is more apparent when we write the optimality conditions given by the maximum principle.

The optimal control problem to solve is

$$
\begin{aligned}
& V^{0}\left(\mathbf{K}^{0}\right) \\
& \quad=\max \int_{0}^{\infty} \mathrm{e}^{-(\rho+\lambda) t}\left\{U(C(t))+\lambda W\left(K_{1}(t), K_{2}(t)\right)\right\} \mathrm{d} t
\end{aligned}
$$

s.t.

$K_{j}(0)=K_{j}^{0}, \quad j=1,2$,

$\dot{K}_{j}(t)=I_{j}(t)-\delta_{j} K_{j}(t), \quad j=1,2$,

$C(t)=f^{0}\left(K_{1}(t)\right)-I_{1}(t)-I_{2}(t)$.

Introduce the current-value hamiltonian

$$
\begin{aligned}
& H(\mathbf{K}, \mathbf{p}, \mu, \mathbf{I}, C) \\
& \quad=U(C)+\lambda W\left(K_{1}, K_{2}\right)+\sum_{j=1,2} p_{j}\left(I_{j}-\delta_{j} K_{j}\right) \\
& \quad+\mu\left(f^{0}\left(K_{1}\right)-I_{1}-I_{2}-C\right)
\end{aligned}
$$

with $p_{j}(t)=\mathrm{e}^{(\rho+\lambda) t} v_{j}(t)$, where $v_{j}(t)$ is the costate variable associated with the dynamics of $K_{j}, j=1,2$ (that are interpreted as marginal utilities of capital stocks) and $\mu$ is the Kuhn-Tucker multiplier ${ }^{5}$ associated with the constraint (23). Omitting, for

5 We always assume that sufficient regularity conditions hold for the $\mathrm{K}-\mathrm{T}$ multiplier to exist. 
Table 1

Signs of marginals

\begin{tabular}{lr}
\hline$f^{0^{\prime}}\left(K_{1}\right)$ & $>0$ \\
$V^{1^{\prime}}\left(K_{1}-\Phi(\mathbf{K})\right)$ & $>0$ \\
$\frac{\partial}{\partial K_{1}} \Phi(\mathbf{K})$ & $>0$ \\
$\frac{\partial}{\partial K_{2}} \Phi(\mathbf{K})$ & $<0$ \\
$\Psi^{\prime}\left(K_{2}\right)$ & $<0$
\end{tabular}

the sake of concision, the time indexing and the function arguments the first-order necessary conditions write as follows:

$\dot{K}_{j}=\frac{\partial}{\partial p_{j}} H, \quad j=1,2$,

$\dot{p}_{j}=-\frac{\partial}{\partial K_{j}} H+(\rho+\lambda) p_{j}, \quad j=1,2$,

$0=\frac{\partial}{\partial I_{j}} H, \quad j=1,2$,

$0=\frac{\partial}{\partial C} H$

Let us focus on the decision (control) variables first. Condition (28) yields

$U^{\prime}(C)=\mu$

while Conditions (27) yield

$p_{j}=\mu, \quad j=1,2$.

So, as usual in these types of models the optimal path equalizes the marginal utilities of the two capital stocks and the marginal utility of consumption. Hence, what is critical is the evolution of the marginal utility of capital stocks. Detailing Eq. (26) for each type of capital stock we get

$\dot{p}_{1}=-\lambda \frac{\partial}{\partial K_{1}} W(\mathbf{K})-\mu f^{0^{\prime}}\left(K_{1}\right)+\left(\rho+\lambda+\delta_{1}\right) p_{1}$,

$\dot{p}_{2}=-\lambda \frac{\partial}{\partial K_{2}} W(\mathbf{K})+\left(\rho+\lambda+\delta_{2}\right) p_{2}$.

Recall that we have defined

$W(\mathbf{K})=V^{1}\left(K_{1}-\Phi(\mathbf{K})\right)-\Psi\left(K_{2}\right)$

so the costate equations can be rewritten with more details

$$
\begin{aligned}
\dot{q}_{1}= & -\lambda V^{1^{\prime}}\left(K_{1}-\Phi(\mathbf{K})\right)\left(1-\frac{\partial}{\partial K_{1}} \Phi(\mathbf{K})\right) \\
& -\mu f^{0^{\prime}}\left(K_{1}\right)+\left(\rho+\lambda+\delta_{1}\right) p_{1}, \\
\dot{q}_{2}= & \lambda\left(V^{1^{\prime}}\left(K_{1}-\Phi(\mathbf{K})\right) \frac{\partial}{\partial K_{2}} \Phi(\mathbf{K})+\Psi^{\prime}\left(K_{2}\right)\right) \\
& +\left(\rho+\lambda+\delta_{2}\right) p_{2} .
\end{aligned}
$$

We may expect the signs of marginal values indicated in Table 1.

\subsubsection{Turnpike}

The catastrophe occurrence is a low-probability event. In practice, the intensity $\lambda$ will be pretty small. Therefore, the expected value of the stopping time $T$ is quite large and the economy will have, with high probability, enough time to reach a quasi-steady state where the capital stocks are maintained constant over time. This is why it is interesting to look at the extremal steady state of this infinite horizon control system. Under relatively general conditions discussed in Carlson et al. (1994) one can expect this steady state, also called turnpike, to be an attractor for all optimal capital accumulation strategies. The extremal steady state is a stationary solution of the first order optimality conditions, i.e. a solution $\left(\bar{\mu}, \bar{C}, \bar{I}_{1}, \bar{I}_{2}, \bar{K}_{1}, \bar{K}_{2}, \bar{p}_{1}, \bar{p}_{2}\right)$ to the following system of algebraic equations:

$0 \leqslant \mu$,

$0=\mu\left(f^{0}\left(K_{1}\right)-C-I_{1}-I_{2}\right)$,

$C=f^{0}\left(K_{1}\right)-I_{1}-I_{2}$,

$\mu=p_{j}, \quad j=1,2$,

$\mu=U^{\prime}(C)$

$0=I_{1}-\delta_{1} K_{1}$,

$0=I_{2}-\delta_{2} K_{2}$,

$$
0=-\lambda V^{1^{\prime}}\left(K_{1}-\Phi(\mathbf{K})\right)\left(1-\frac{\partial}{\partial K_{1}} \Phi(\mathbf{K})\right)
$$$$
-\mu f^{0^{\prime}}\left(K_{1}\right)+\left(\rho+\lambda+\delta_{1}\right) p_{1} \text {, }
$$

$0=\lambda\left(V^{1^{\prime}}\left(K_{1}-\Phi(\mathbf{K})\right) \frac{\partial}{\partial K_{2}} \Phi(\mathbf{K})+\Psi^{\prime}\left(K_{2}\right)\right)$ $+\left(\rho+\lambda+\delta_{2}\right) p_{2}$.

It has been shown (Carlson et al., 1994) that this set of equations is also the first-order optimality conditions of the implicit programming problem:

$\max \quad U(C)+\lambda W(\mathbf{K})$

s.t.

$0=I_{1}-\delta_{1} K_{1}-(\rho+\lambda)\left(K_{1}-\bar{K}_{1}\right)$,

$0=I_{2}-\delta_{2} K_{2}-(\rho+\lambda)\left(K_{2}-\bar{K}_{2}\right)$,

$C=f^{0}\left(K_{1}\right)-I_{1}-I_{2}$,

where $\overline{\mathbf{K}}=\left(\bar{K}_{1}, \bar{K}_{2}\right)$ is itself the solution of (44)-(47). Therefore, the computation of the turnpike involves a fixed point calculation.

\subsubsection{A note on existence of solutions}

This model is an instance of piecewise deterministic control systems studied in Davis (1984) and Vermes (1985) where existence conditions are given. The turnpike property for this class of systems has been studied in Fleming et al. (1987), Haurie and Van Delft (1995) and Carlson et al. (1994). 


\subsection{The case of an anthropogenic influence on the catastrophe process}

Let us consider now the case where the catastrophe process has a probability measure which is influenced by a stock of emissions that are associated with the production process, for example, the concentration $M(t)$ of $\mathrm{CO}_{2}$ accumulated at time $t$ in the atmosphere.

The economy dynamics is now characterized by the productive capital accumulation equations and also the $\mathrm{CO}_{2}$ accumulation equation

$\dot{K}_{1}(t)=I_{1}(t)-\delta_{1} K_{1}(t)$,

$\dot{K}_{2}(t)=I_{2}(t)-\delta_{2} K_{2}(t)$,

$\dot{M}(t)=g(M(t), E(t))$,

where $E(t)$ is the emission rate of $\mathrm{CO}_{2}$ by the production sector and $g(\cdot, \cdot)$ is the net $\mathrm{CO}_{2}$ accumulation rate. As in Nordhaus (1994) we assume that the emissions are defined by

$E(t)=\sigma(1-v(t)) Y(t)$,

where $\sigma$ is the initial carbon intensity of the economy and $v(t) \in$ $[0,1]$ is the abatement effort at time $t$. Also, as in Nordhaus (1994), we assume that the emission abatement effort has a cost expressed in output loss, that is the following must hold:

$C(t)+I_{1}(t)+I_{2}(t)=D(v(t)) Y(t)$,

where $D(v(t)) \in[0,1]$ is the production loss function associated with the abatement effort. ${ }^{6}$

We assume that the occurrence of the catastrophe is well described as a jump process $\{\zeta(t): t \geqslant 0\}$ with jump rate

$q(M(t))=\lim _{\mathrm{d} t \rightarrow 0} \frac{\mathrm{P}[\zeta(t+\mathrm{d} t)=1 \mid \zeta(t)=0, M(t)]}{\mathrm{d} t}$,

where the function $q(M)$ is monotone nondecreasing in $M$. The rest of the model is similar to the previous case. ${ }^{7}$

\subsubsection{Optimal capital and $\mathrm{CO}_{2}$ accumulation path}

The problem of the optimal accumulation of capitals $K_{1}, K_{2}$ and pollution $M$ is formulated as in section 2.1.2. After the occurrence of the catastrophe, the problem for the surviving economy is the same and can be summarized by the welfare function $W\left(K_{1}, K_{2}\right)$. The only difference comes from the dependency of the jump process probability measure on the concentrations $M(t)$. Repeating the same integration by parts we obtain that the initial welfare value function is given by

$$
\begin{aligned}
V^{0}\left(\mathbf{K}^{0}, M^{0}\right)= & \int_{0}^{\infty} \mathrm{e}^{-\left(\rho t+\int_{0}^{t} q(M(s)) \mathrm{d} s\right)}\{U(C(t)) \\
& \left.+q(M(t)) W\left(K_{1}(t), K_{2}(t)\right)\right\} \mathrm{d} t .
\end{aligned}
$$

6 The reader will notice that we did not introduce a loss of output directly linked with the pollution stock variable, or with a climate variable, like temperature that is influenced by $M$. This choice has been made to keep the model focussed on the catastrophe prevention issue.

7 For the sake of keeping the simplest form of the model, we have assumed that the GHG concentration $M$ is influencing the jump rate. It would be easy to introduce a temperature $T$ state equation and to assume that $q(\cdot)$ depends on $T$ instead of $M$.
This defines an infinite horizon control problem with an endogenous discount rate given by $\rho+q(M(t))$.

\subsubsection{Interpretation of optimality conditions}

The optimal control problem to solve is

$$
\begin{aligned}
V^{0}\left(\mathbf{K}^{0}, M^{0}\right)= & \max \int_{0}^{\infty} \mathrm{e}^{-\left(\rho t+\int_{0}^{t} q(M(s)) \mathrm{d} s\right)}\{U(C(t)) \\
& \left.+q(M(t)) W\left(K_{1}(t), K_{2}(t)\right)\right\} \mathrm{d} t .
\end{aligned}
$$

s.t.

$K_{j}(0)=K_{j}^{0}, \quad j=1,2$,

$M(0)=M^{0}$,

$\dot{K}_{j}(t)=I_{j}(t)-\delta_{j} K_{j}(t), \quad j=1,2$,

$\dot{M}(t)=g(M(t), E(t))$

$E(t)=\sigma(1-v(t)) f^{0}\left(K_{1}(t)\right)$,

$C(t)=D(v(t)) f^{0}\left(K_{1}(t)\right)-I_{1}(t)-I_{2}(t)$,

$v(t) \in[0,1]$.

To deal with the endogenous discount rate we have to introduce a new state variable $z(t)$ with state equation $\dot{z}(t)=$ $q(M(t))$. The optimal control problem to solve is then reformulated as follows:

$\begin{aligned} V^{0}\left(\mathbf{K}^{0}, M^{0}\right)= & \max \int_{0}^{\infty} \mathrm{e}^{-(\rho t+z(t))}\{U(C(t)) \\ & \left.+q(M(t)) W\left(K_{1}(t), K_{2}(t)\right)\right\} \mathrm{d} t .\end{aligned}$

s.t.

$K_{j}(0)=K_{j}^{0}, \quad j=1,2$,

$M(0)=M^{0}$,

$\dot{K}_{j}(t)=I_{j}(t)-\delta_{j} K_{j}(t), \quad j=1,2$,

$\dot{M}(t)=g(M(t), E(t))$,

$E(t)=\sigma(1-v(t)) f^{0}\left(K_{1}(t)\right)$,

$C(t)=D(v(t)) f^{0}\left(K_{1}(t)\right)-I_{1}(t)-I_{2}(t)$,

$v(t) \in[0,1]$

$\dot{z}(t)=q(M(t))$.

Introduce the current-value hamiltonian

$$
\begin{aligned}
& H(\mathbf{K}, M, \mathbf{p}, r, \mu, v, \mathbf{I}, C, E, v) \\
& =U(C)+q(M) W\left(K_{1}, K_{2}\right)+\sum_{j=1,2} p_{j}\left(I_{j}-\delta_{j} K_{j}\right) \\
& \quad+r g(M, E)+\mu\left(D(v) f^{0}\left(K_{1}\right)-I_{1}-I_{2}-C\right) \\
& \quad+v\left(\sigma(1-v) f^{0}\left(K_{1}\right)-E\right)+\pi q(M),
\end{aligned}
$$

with $p_{j}(t)=\mathrm{e}^{(\rho t+z(t))} \vartheta_{j}(t)$, where $\vartheta_{j}(t)$ is the costate variable associated with the dynamics of $K_{j}, j=1,2$ (that are interpreted as marginal utilities of capital stocks), $r=\mathrm{e}^{(\rho t+z(t))} \varpi(t)$, 
where $\varpi(t)$ is the costate variable associated with the $\mathrm{CO}_{2}$ accumulation equation (67) and $\mu$ and $v$ are the Kuhn-Tucker multipliers associated with the constraint (69) and (68), respectively. Finally, $\pi(t)=\mathrm{e}^{(\rho t+z(t))} \omega(t)$ where $\omega(t)$ is the costate variable associated with the auxiliary state equation (71).

The first-order necessary conditions write as follows:

$\dot{K}_{j}=\frac{\partial}{\partial p_{j}} H, \quad j=1,2$,

$\dot{M}=\frac{\partial}{\partial r} H$,

$\dot{p}_{j}=-\frac{\partial}{\partial K_{j}} H+(\rho+q(M)) p_{j}, \quad j=1,2$,

$\dot{r}=-\frac{\partial}{\partial M} H+(\rho+q(M)) r$,

$\dot{\pi}=U(C)+q(M) W\left(K_{1}, K_{2}\right)+(\rho+q(M)) \pi$,

$0=\frac{\partial}{\partial I_{j}} H, \quad j=1,2$,

$0=\frac{\partial}{\partial C} H$,

$0=\frac{\partial}{\partial E} H$,

$0=\frac{\partial}{\partial v} H$,

$E=\sigma(1-v) f^{0}\left(K_{1}\right)$

$C=D(v) f^{0}\left(K_{1}\right)-I_{1}-I_{2}$,

$0 \leqslant \mu$,

$0=\mu\left(D(v) f^{0}\left(K_{1}\right)\right)-I_{1}-I_{2}-C$.

Focusing on the control variable we get the following conditions:

$U^{\prime}(C)=\mu$,

while Conditions (78) yield

$p_{j}=\mu, \quad j=1,2$.

Condition (80) yields

$v=r \frac{\partial}{\partial E} g(M, E)$,

and, finally Condition (81) gives

$v \sigma=\mu D^{\prime}(v)$.

Conditions (86)-(87) have already been interpreted. Condition (88) says that the marginal cost of the contribution of emission $E$ to concentration should be equal to the marginal cost of the emission process associated to production. Finally, Condition (89) tells that the marginal value loss associated with the marginal production loss due to abatement should be equal to the marginal value of abatement.

\subsubsection{Asymptotic steady state}

A steady state for the optimal control problem defined above will be given by a set of values $(\overline{\mathbf{K}}, \bar{M}, \overline{\mathbf{p}}, \bar{r}, \bar{\mu}, \bar{v}, \overline{\mathbf{I}}, \bar{C}, \bar{E}, \bar{v})$ that satisfy the first order stationary conditions where all time derivatives are replaced by 0 .

$0=\frac{\partial}{\partial p_{j}} H, \quad j=1,2$,

$0=\frac{\partial}{\partial r} H$,

$0=-\frac{\partial}{\partial K_{j}} H+(\rho+q(M)) p_{j}, \quad j=1,2$,

$0=-\frac{\partial}{\partial M} H+(\rho+q(M)) r$,

$0=U(C)+q(M) W\left(K_{1}, K_{2}\right)+(\rho+q(M)) \pi$,

$0=\frac{\partial}{\partial I_{j}} H, \quad j=1,2$,

$0=\frac{\partial}{\partial C} H$,

$0=\frac{\partial}{\partial E} H$,

$0=\frac{\partial}{\partial v} H$

$E=\sigma(1-v) f^{0}\left(K_{1}\right)$,

$C=D(v)\left(f^{0}\left(K_{1}\right)-I_{1}-I_{2}\right)$,

$0 \leqslant \mu$,

$0=\mu\left(D(v)\left(f^{0}\left(K_{1}\right)\right)-I_{1}-I_{2}-C\right.$.

\subsubsection{A note on existence of solutions}

This model is also a particular instance of piecewise deterministic control systems studied in Davis (1984) and Vermes (1985) and conditions for existence of solutions can be easily checked. However, the turnpike property is much harder to establish. We will thus rely on numerical simulations to check that this property holds for that model too.

\subsection{How to deal with intergenerational equity}

\subsubsection{Adjusting the pure rate of time preference}

The optimal economic growth with catastrophe prevention pauses interesting problems of time scaling. In practice, the very low jump rate characterizing the catastrophe process might be several orders of magnitude smaller than the pure time preference discount rate; for example $\rho=3 \%$ is usually adopted for $\mathrm{CBA}^{8}$ related to global warming, whereas $\lambda=0.00000001$ is the intensity for an asteroid collision. ${ }^{9}$ Even in the case of an

\footnotetext{
${ }^{8}$ Cost-benefit analysis.

${ }^{9}$ One counts one big hit for 100000000 years.
} 
anthropogenic influence on the catastrophe occurrence rate, one may expect this order of magnitude difference between $\rho=3 \%$ and $q(\bar{M})$ which is of the order of 0.005 .

We may view the discounting and the catastrophe processes as two independent jump processes that evolve independently and in parallel. The discounting process represents a life duration for the consuming agent described by an exponential random variable with expected length of life $1 / \rho$. The expected time to the catastrophe event is given by $1 / \lambda$ or approximately by $1 / q(\bar{M})$. It should be clear that a decision maker using a high discount rate $\rho$ will not be concerned with a catastrophic event having a very low occurrence rate $\lambda$ or $q(\bar{M})$. Indeed one could reduce the discount rate $\rho$ to a value that is commensurate with $q(\bar{M})$. This would mean that the planner is interested in a (random) planning horizon that may include with good probability of the time of occurrence of the catastrophe. However, when one decreases the pure time preference rate $\rho$ one generates a value function which tends to be more "flat", hence with a smaller sensitivity to initial values of state variables. This implies that the cost of capital losses will be reduced.

\subsubsection{Introducing a direct concern for the post-catastrophe generation}

We may introduce, in the utility function of the present generation (which has a high probability of not witnessing the catastrophe), a direct concern for the welfare of the generations that will follow as its descendents. In Haurie (to appear), Haurie and Moresino (to appear) a multigeneration stochastic game model has been proposed. It has been shown that if we assume that each generation $\ell$ lives between times $t^{\ell}$ and $t^{\ell+1}$ with an exponentially distributed life duration of expected value $1 / \rho$ and if each generation $k$ has a utility function

$U_{k}(\gamma)=\sum_{\ell \geqslant k} \beta^{\ell-k} \mathrm{E}_{\gamma}\left[\int_{t^{\ell}}^{t^{\ell+1}} U(C(t)) \mathrm{d} t\right]$,

where $0 \leqslant \beta<1$ and where $\gamma$ is the intergenerational equilibrium policy, this is equivalent to having the current generation use a discount rate $\rho(1-\beta)$ in its CBA exercise. Notice that (103) implies that the current generation $k$ puts a weight $\beta^{\ell-k}$ on the welfare of forthcoming generation $\ell$. We refer the reader to Haurie (to appear), Haurie and Moresino (to appear) for more details on this game theoretic justification for using low discount rates in CBA. In the next section we explore via numerical methods the impact of the relative magnitudes of discount and jump rates in the long-term behavior of this economic growth process.

\section{Numerical solutions}

In this section, we compute the turnpike values for both models presented in Section 2 and for different parameters values. We use a numerical method inspired by Kushner and Dupuis (KD) (see Kushner \& Dupuis, 1992) to approximate the optimal policy. We also implement a direct method for computing turnpikes by solving the steady-state necessary conditions. Comparing these results we can check numerically that the model with controlled jump (catastrophe) rate also admits a turnpike.

\subsection{Solving the stochastic control problem via KD method}

In this subsection, we provide the Hamilton-Jacobi-Belmann (HJB) system of equations associated with the controlled catastrophe model. We then show how the HJB system of equations can be approximated by a Markov decision problem (MDP) using KD method.

\subsubsection{The HJB system of equations}

Let us first recall the problem we want to solve

$$
\begin{aligned}
V^{0}(x)= & \max E\left[\int_{0}^{T} \mathrm{e}^{-\rho t} L^{0}(x, u) \mathrm{d} t\right. \\
& \left.-\mathrm{e}^{-\rho T}\left(V^{1}(x(T)-\Phi(x(T)))-\Psi(x(T))\right)\right], \\
V^{1}(x)= & \max \left[\int_{0}^{\infty} \mathrm{e}^{-\rho t} L^{1}(x, u) \mathrm{d} t\right],
\end{aligned}
$$

with

$\dot{x}=\tilde{f}^{j}(x, u), \quad j=0,1$.

Applying standard dynamic programming analysis, we obtain the following set of HJB equations that provide sufficient optimality conditions for this stochastic control problem (Fleming \& Rishel, 1975). In full generality we should invoke viscosity solutions, although the economic growth models are known to provide smooth value functions.

$$
\begin{aligned}
\rho V^{0}(x)= & \max _{u}\left\{L^{0}(x, u)+\frac{\partial}{\partial x} V^{0}(x) \tilde{f}^{0}(x, u)\right. \\
& \left.+\left[V^{1}(x-\Phi(x))-\Psi(x)-V^{0}(x)\right] q(x)\right\}
\end{aligned}
$$

and

$\rho V^{1}(x)=\max _{u}\left\{L^{1}(x, u)+\frac{\partial}{\partial x} V^{1}(x) \tilde{f}^{1}(x, u)\right\}$.

\subsubsection{The approximating $M D P$}

To simplify notation omit the superscript $j$ and write $\tilde{f}^{j}(x, u)=\tilde{f}(x, u)$. We approximate the partial derivatives as follows:

$\frac{\partial}{\partial x_{i}} V^{j}(x) \rightarrow \begin{cases}\left(V^{j}\left(x+e_{i} h_{i}\right)-V^{j}(x)\right) / h_{i} & \text { if } \tilde{f}_{i}(x, u) \geqslant 0, \\ \left(V^{j}(x)-V^{j}\left(x-e_{i} h_{i}\right)\right) / h_{i} & \text { if } \tilde{f}_{i}(x, u)<0,\end{cases}$

where $e_{i}$ is the unit vector of the $i$ th axis. Define

$\tilde{f}_{i}^{+}(x, u)=\max \left\{0, \tilde{f}_{i}(x, u)\right\}$,

$\tilde{f}_{i}^{-}(x, u)=\max \left\{0,-\tilde{f}_{i}(x, u)\right\}$. 
Substituting the differences to the partial derivatives in Equation (107), one obtains

$$
\begin{aligned}
\rho V^{0}(x)= & \max _{u \in U(x)}\left\{L^{1}(x, u)\right. \\
& +\left[V^{1}(x-\Phi(x))-\Psi(x)-V^{0}(x)\right] q(x) \\
& +\sum_{i=1}^{n}\left(\frac{\left(V^{0}\left(x+e_{i} h_{i}\right)-V^{0}(x)\right)}{h_{i}} \tilde{f}_{i}^{+}(x, u)\right. \\
& \left.\left.+\frac{\left(V^{0}(x)-V^{0}\left(x-e_{i} h_{i}\right)\right)}{h_{i}} \tilde{f}_{i}^{-}(x, u)\right)\right\} .
\end{aligned}
$$

Define the interpolation intervals

$$
\begin{aligned}
& \Delta(x, 0, u)=\frac{1}{\rho+q(x)+\sum_{i=1}^{n}\left(\left|\tilde{f}_{i}(x, u)\right|\right) / h_{i}}, \\
& \Delta(x, 1, u)=\frac{1}{\rho+\sum_{i=1}^{n}\left(\left|\tilde{f}_{i}(x, u)\right|\right) / h_{i}} .
\end{aligned}
$$

One considers an MDP with discrete states $x^{g}, g \in$ $\mathscr{G}$ and control $u \in U\left(x^{g}\right)$. The transition rewards are given by $L^{j}\left(x^{g}, u\right) \Delta(x, j, u)$. The transition probabilities $\Pi\left(x^{g}, x^{g^{\prime}}, j, j^{\prime}, u\right)$ are defined as follows:

- When $g \in \mathscr{G} \backslash \partial \mathscr{G}$ the transition probabilities from $x^{g}$ to any neighboring sampled value $x^{g} \pm e_{i} h_{i}$, if we stay in state $j=0$, are given by

$$
\Pi\left(x^{g}, x^{g} \pm e_{i} h_{i}, 0,0, u\right)=\frac{\left(\tilde{f}_{i}^{ \pm}\left(x^{g}, u\right)\right) / h_{i}}{q\left(x^{g}\right)+\sum_{j=1}^{n}\left(\left|\tilde{f}_{j}\left(x^{g}, u\right)\right|\right) / h_{j}}
$$

and providing that we stay in state $j=1$ they are given by

$$
\Pi\left(x^{g}, x^{g} \pm e_{i} h_{i}, 1,1, u\right)=\frac{\left(\tilde{f}_{i}^{ \pm}\left(x^{g}, u\right)\right) / h_{i}}{\sum_{j=1}^{n}\left(\left|\tilde{f}_{j}\left(x^{g}, u\right)\right|\right) / h_{j}} .
$$

- Define $x^{+}=\min \left\{x^{g^{\prime}} \mid g^{\prime} \in \mathscr{G}, x^{g^{\prime}} \geqslant x^{g}-\Phi\left(x^{g}\right)\right\}$ and $x^{-}=$ $\max \left\{x^{g^{\prime}} \mid g^{\prime} \in \mathscr{G}, x^{g^{\prime}} \leqslant x^{g}-\Phi\left(x^{g}\right)\right\}$. If $x^{+}=x^{-}$, the transition probabilities from $j=0$ to $j=1$, are given by

$$
\Pi\left(x^{g}, x^{+}, 0,1, u\right)=\frac{q\left(x^{g}\right)}{q\left(x^{g}\right)+\sum_{j=1}^{n}\left(\left|\tilde{f}_{j}\left(x^{g}, u\right)\right|\right) / h_{j}} .
$$

If $x^{+} \neq x^{-}$, we have

$$
\begin{aligned}
& \Pi\left(x^{g}, x^{+}, 0,1, u\right) \\
& \quad=\frac{x^{g}-\Phi\left(x^{g}\right)-x^{-}}{x^{+}-x^{-}} \frac{q\left(x^{g}\right)}{q\left(x^{g}\right)+\sum_{j=1}^{n}\left(\left|\tilde{f}_{j}\left(x^{g}, u\right)\right|\right) / h_{j}}, \\
& \Pi\left(x^{g}, x^{-}, 0,1, u\right) \\
& \quad=\frac{x^{+}-x^{g}+\Phi\left(x^{g}\right)}{x^{+}-x^{-}} \frac{q\left(x^{g}\right)}{q\left(x^{g}\right)+\sum_{j=1}^{n}\left(\left|\tilde{f}_{j}\left(x^{g}, u\right)\right|\right) / h_{j}} .
\end{aligned}
$$

The transition probabilities for a jump from $j=1$ to 0 are zero.
- On the boundary $\partial \mathscr{G}$ of the grid, the probabilities are defined according to a reflecting boundary scheme.

- All the other transition probabilities are zero.

Discounting factors are defined by

$$
\begin{aligned}
& \beta\left(x^{g}, 0, u\right)=\Delta(x, 0, u)\left(q(x)+\sum_{i=1}^{n} \frac{\left|\tilde{f}_{i}\left(x^{g}, u\right)\right|}{h_{i}}\right), \\
& \beta\left(x^{g}, 1, u\right)=\Delta(x, 1, u)\left(\sum_{i=1}^{n} \frac{\left|\tilde{f}_{i}\left(x^{g}, u\right)\right|}{h_{i}}\right) .
\end{aligned}
$$

The DP equations for this approximating MDP are given by

$$
\begin{aligned}
v^{0}\left(x^{g}\right)= & \max _{u \in U\left(x^{g}\right)}\left\{L^{0}\left(x^{g}, u\right) \Delta(x, 0, u)\right. \\
& +\beta\left(x^{g}, 0, u\right) \sum_{g^{\prime} \in \mathscr{G}} \Pi\left(x^{g}, x^{g^{\prime}}, 0,1, u\right) v^{j}\left(x^{g^{\prime}}\right) \\
& \left.-\beta\left(x^{g}, 0, u\right) \sum_{x^{g^{\prime}} \in\left\{x^{+}, x^{-}\right\}} \Pi\left(x^{g}, x^{g^{\prime}}, 0,1, u\right) \Psi\left(x^{g}\right)\right\},
\end{aligned}
$$

$$
\begin{aligned}
v^{1}\left(x^{g}\right)= & \max _{u \in U\left(x^{g}\right)}\left\{L^{1}\left(x^{g}, u\right) \Delta(x, 1, u)\right. \\
& \left.+\beta\left(x^{g}, 1, u\right) \sum_{g^{\prime} \in \mathscr{G}} \Pi\left(x^{g}, x^{g^{\prime}}, 1,1, u\right) v^{1}\left(x^{g^{\prime}}\right)\right\} .
\end{aligned}
$$

To solve both DP equations (114) and (115) one uses a linear programming formulation, as indicated in Puterman (1994, p. 223). It is well established that the approximating MDPs lead to approximations of the value functions that converge weakly toward the continuous time solutions of the DP equations. Using the classical verification theorems of DP, we conclude that the optimal solutions obtained from the approximating MDPs provide an $\varepsilon$-optimal solutions to the continuous time problem.

\subsection{Computing turnpikes for the uncontrolled catastrophe model}

A numerical experimentation can be made, using an IAM ${ }^{10}$ like DICE94 (see Nordhaus, 1994 or ICLIPS. ${ }^{11}$ These models refer to the whole word, and the catastrophe we have in mind would also have a global effect that is described in the model by the functions $\Psi\left(K_{2}\right)$ and $\Phi\left(K_{1}, K_{2}\right)$. The catastrophe rate function $\lambda$ has also to be calibrated. The solution of the model with $\zeta=1$, gives an evaluation of the function $V^{1}\left(K_{1}\right)$.

\footnotetext{
${ }^{10}$ Integrated assessment model.

11 This model has been used in particular for the implementation of the Tolerable Windows Approach (Petschel-Held, Schellnhuber, Bruckner, Toth, \& Hasselmann, 1999)
} 
Table 2

Parameters values

\begin{tabular}{lcll}
\hline$\delta_{1}$ & 0.1 & $\delta_{2}$ & 0.1 \\
$\bar{L}$ & 12000 & $\bar{A}$ & 0.063 \\
$v_{1}$ & 20 & $v_{2}$ & 0.1 \\
$\ell_{1}$ & 0.90 & $\ell_{2}$ & 0.0003 \\
$\gamma$ & 0.25 & & \\
\hline
\end{tabular}

This is the value function of a pure Ramsey-Cass economic growth model. We can solve the Ramsey problem from different initial state values $K_{1}^{i}$ and fit a functional form to these estimates. The solution of the model with $\zeta=0$ will then be looked for.

We show below a numerical model, inspired from DICE94. The values $\bar{L}$ and $\bar{A}$ represent the asymptotic values of population and technological progresses in this model.

$\max \int_{0}^{\infty} \mathrm{e}^{-\rho t} \log (C(t)) \mathrm{d} t$,

$\dot{L}(t)=g_{L}(t) L(t)$,

$\dot{g}_{L}(t)=-\delta_{L} g_{L}(t)$

$\dot{A}(t)=g_{A}(t) A(t)$,

$\dot{g}_{A}(t)=-\delta_{A} g_{A}(t)$,

$Y(t)=A(t) K_{1}(t)^{\gamma} L(t)^{1-\gamma}$

$$
=C(t)+I_{1}(t)+I_{2}(t),
$$

$\dot{K}_{j}(t)=I_{j}(t)-\delta_{j} K(t), \quad j=1,2$.

We assume the following form for the damage functions:

$$
\begin{aligned}
& \Psi\left(K_{2}\right)=v_{1} /\left(1+v_{2} \sqrt{K_{2}}\right), \\
& \Phi\left(K_{1}, K_{2}\right)=\ell_{1} K_{1} /\left(1+\log \left(1+\ell_{2} K_{2}\right)\right) .
\end{aligned}
$$

According to the parameter values shown in Table 2 the capital destruction rate at a catastrophe occurrence is $90 \%$ when there is no preventive capital available. Also the catastrophe cost is in the range of 20 when $K_{2}=0$ which is around $20 \%$ of the utility gained from the consumption in the infinite horizon economy. The value function in mode $\zeta=1$, for $\rho=0.03$, respectively $\rho=0.06$, is given in (126) and in (127), respectively. It has been obtained in two steps. First, we obtained an approximation of the value function by solving the Ramsey growth model with the numerical method described in Section 3.1. Then, we fit this discrete approximating value function with an analytical function. In our case we have an excellent least-square fit $R^{2}=$ 0.999999998

$$
\begin{aligned}
V^{1}(K)= & 185.771751-0.310653189 K^{0.6} \\
& +1.850646784 K^{0.5} \\
& -2.949629208 K^{0.4}+1.670241443 K^{0.3},
\end{aligned}
$$

$V^{1}(K)=91.12230248-0.308780416 K^{0.6} 1.755896839 K^{0.5}$

$$
-2.715196395 K^{0.4} 1.500248749 K^{0.3} \text {. }
$$

Table 3

Turnpike values for $\zeta=0$ and different risk rate values

\begin{tabular}{lllrl}
\hline$\rho$ & $\lambda$ & $K_{1}$ & \multicolumn{1}{c}{$K_{2}$} & \multicolumn{1}{l}{$C$} \\
\hline 0.03 & $1 \times 10^{-6}$ & 719.40 & 0.00 & 302.14 \\
0.03 & $1 \times 10^{-3}$ & 704.52 & 2.78 & 301.40 \\
0.03 & $5 \times 10^{-3}$ & 649.18 & 22.58 & 297.42 \\
0.03 & $1 \times 10^{-2}$ & 586.88 & 45.17 & 292.31 \\
0.06 & $1 \times 10^{-6}$ & 545.41 & 0.00 & 294.52 \\
0.06 & $1 \times 10^{-3}$ & 535.97 & 1.94 & 293.75 \\
0.06 & $5 \times 10^{-3}$ & 500.01 & 17.48 & 289.81 \\
0.06 & $1 \times 10^{-2}$ & 459.12 & 36.50 & 284.79 \\
\hline
\end{tabular}

Table 4

Parameters values

\begin{tabular}{lclllcll}
\hline$\sigma$ & 0.33 & $\delta_{1}$ & 0.1 & $\delta_{2}$ & 0.1 & $\delta_{M}$ & 0.0833 \\
$\beta$ & 0.64 & $\gamma$ & 0.25 & $\bar{L}$ & 12000 & $\bar{A}$ & 0.063 \\
$v_{1}$ & 20 & $v_{2}$ & 0.1 & $\ell_{1}$ & 0.95 & $\ell_{2}$ & 0.002 \\
$b_{1}$ & 0.045 & $b_{2}$ & 2.15 & & & & \\
\hline
\end{tabular}

\subsection{Turnpikes for different risk rate values}

The turnpike values are computed by solving directly the implicit programming problem (44)-(47) with EXCEL's solver. Table 3 gives the results of our calculations concerning the turnpike levels in mode $\zeta=0$, when the risk rate of catastrophe is $0.001 \%, 0.005 \%$ and $0.01 \%$, respectively. We can see how the increase in the catastrophe risk rate $\lambda$ changes the values of the preventive capital stock that is maintained at an optimal steady state in mode $\zeta=0$. We can see also that the steady-state productive capital stock and the value of consumption decrease when the catastrophe risk increases.

\subsection{Computing turnpikes for the controlled catastrophe model}

We report below on the numerical solutions obtained, using the same adaptation of DICE94 as above, for the turnpike values in mode $\zeta=0$, when the accumulation of GHGs is described by the equation

$\dot{M}(t)=\beta E(t)-\delta_{M}(M(t)-590)$,

whereas the catastrophe risk rate is an affine function of the concentration $M(t)$

$q(M(t))=\eta_{1}+\eta_{2} M(t)$.

Emissions $E(t)$ are given by

$E(t))=(1-v(t)) \sigma Y(t)$

where $v(t) \in[0,1]$ is the abatement effort and $\sigma$ is the nominal emission rate per unit of output. One assumes that the abatement cost is represented by a loss of output given by the function

$\Omega(t))=\left(1-b_{1} v(t)^{b_{2}}\right)$.

Table 4 gives the values of the parameters introduced in Eqs. (128)-(131). 
Table 5

Turnpike values for $\zeta=0$ and different risk rate values

\begin{tabular}{llllrlll}
\hline$\rho$ & $\eta_{1}$ & $\eta_{2}$ & $K_{1}$ & \multicolumn{1}{c}{$K_{2}$} & \multicolumn{1}{l}{$M$} & $v$ & $C$ \\
\hline 0.03 & $10^{-6}$ & $1 \times 10^{-9}$ & 719.36 & 0.00 & 1538.03 & 0.00 & 302.14 \\
0.03 & $10^{-3}$ & $1 \times 10^{-6}$ & 698.07 & 11.11 & 1409.06 & 0.13 & 300.15 \\
0.03 & $10^{-3}$ & $5 \times 10^{-6}$ & 664.36 & 41.03 & 1179.94 & 0.37 & 294.28 \\
0.03 & $10^{-3}$ & $1 \times 10^{-5}$ & 642.09 & 69.80 & 1051.49 & 0.50 & 288.74 \\
0.06 & $10^{-6}$ & $1 \times 10^{-9}$ & 545.40 & 0.00 & 1474.72 & 0.00 & 294.52 \\
0.06 & $10^{-3}$ & $1 \times 10^{-6}$ & 532.23 & 7.67 & 1374.63 & 0.11 & 292.82 \\
0.06 & $10^{-3}$ & $5 \times 10^{-6}$ & 508.92 & 30.02 & 1169.10 & 0.33 & 287.72 \\
0.06 & $10^{-3}$ & $1 \times 10^{-5}$ & 492.55 & 51.33 & 1022.34 & 0.50 & 282.46 \\
\hline
\end{tabular}

Table 6

Space discretization

\begin{tabular}{lrcc}
\hline Space & Minimum & Maximum & Step \\
\hline$K_{1}$ & 0 & 900 & 50 \\
$K_{2}$ & 0 & 20 & 2.5 \\
$M$ & 1300 & 1600 & 100 \\
$I_{1}$ & 0 & 90 & 5 \\
$I_{2}$ & 0 & 2 & 0.25 \\
$\mu$ & 0 & 0.2 & 0.05 \\
\hline
\end{tabular}

Using the software MAPLE, we computed the turnpike values by solving the system of Eqs. (90)-(102) with Newton's method. Note that the value functions in mode $\zeta=1$ are the same as the ones of the uncontrolled model and are given in Eqs. (126) and (127).

In Table 5 the values satisfying the necessary conditions for a turnpike are reported. We see that when the risk rate decreases the steady-state economy in initial mode $\zeta=0$ has a higher capital stock for production and a lower one for prevention and it consumes more. A higher risk rate brings indeed a much higher abatement effort.

\subsection{Numerical solution of the controlled catastrophe model via KD method}

To solve the approximating MDP (Eqs. (114) and (115)), we used AMPL (see Fourer, Gay, \& Kernighan, 1993) coupled with the solver Mosek. The values for the space discretization are given in Table 6 . We then compute an approximating optimal policy by linear interpolation of the discrete point policy of the MDP. To compute the optimal trajectory, we discretize time into small period. At the beginning of each period, we apply the approximating optimal policy and maintain the control constant over that period. Two trajectories with different initial state $\left(K_{1}(0)=850, K_{2}(0)=12, M(0)=1550\right.$ and $K_{1}(0)=650$, $\left.K_{2}(0)=8, M(0)=1450\right)$ for the case when $\rho=3 \%, \eta_{1}=10^{-3}$ and $\eta_{2}=10^{-6}$ are displayed in Fig. 1 . We see distinctly that, whatever the initial point, the trajectories converge to an asymptotic value. Table 7 shows the asymptotic attractors for different values of $\eta_{2}$ when $\eta_{1}=10^{-3}$ and $\rho=3 \%$. To have an idea of the accuracy of our numerical method, we can compare these results with the one from Table 5. We see that our crude approximation gives results that remain comparable with those computed directly from the steady-state necessary conditions. It is important to note that a much finer discretization was not possible due to the curse of dimensionality. This provides however a numerical verification of the existence of a turnpike property for this type of model.

\section{Conclusion}

In this essay a model has been proposed to find an optimal capital accumulation path in an economy facing the prospect of a possible environmental catastrophe. The model follows the general philosophy of CBA as it integrates the expected costs induced by the catastrophe and the cost of investing and maintaining a preventive capital into a global welfare optimization scheme. Technically, the optimal accumulation path is determined by an adjusted discount rate which depends on the intensity of the catastrophe process and also by the introduction of an endogenously defined valuation of capital stocks in the reward rate function of the associated optimal growth problem. The early occurrence of the catastrophe having a low probability, it is expected that the economy will reach a steady state. Conditions characterizing the steady state have been provided. The consideration of these extremal steady states are interesting for the assessment of optimal prevention policy since they indicate how much preventive capital a society should be ready to maintain to prepare for the possible catastrophic event. When the catastrophe is induced by an anthropogenic effect, like the accumulation of GHGs, the analysis could also serve to define the level of emission abatement that the current society should implement.

The issue of time scale discrepancy, when the discount rate and the jump rate (intensity) are different by an order of magnitude, pauses also an important problem for this kind of analysis. A way to adjust the CBA analysis so that it shows concerns for what will impact forthcoming generations has been proposed in Haurie (2005) and Haurie (2006). The model would be based then on a stochastic multigeneration game structure, where the pure time preference (discount) rate $\rho$ is linked with the expected life duration of a generation, whereas each generation introduces in its utility function a concern for the wellbeing of future generations. A multigeneration equilibrium can be computed, again using a dynamic programming argument. A new turnpike would then be defined that would pay more attention to the long-term consequences of accumulating and maintaining a preventive capital. Numerical methods have to be implemented to explore the consequences of these different approaches in tackling with this difficult issue: how to deal with low probability-high cost global environmental events. For that purpose, the approach that has been sketched in this paper should be implemented with a more detailed economic growth model, a more precise carbon cycle description to represent the accumulation of GHGs and a better representation of the influence of the GHG concentration on the catastrophe risk rate. Indeed, a good description of the loss functions when the catastrophe occurs is also to be provided. 

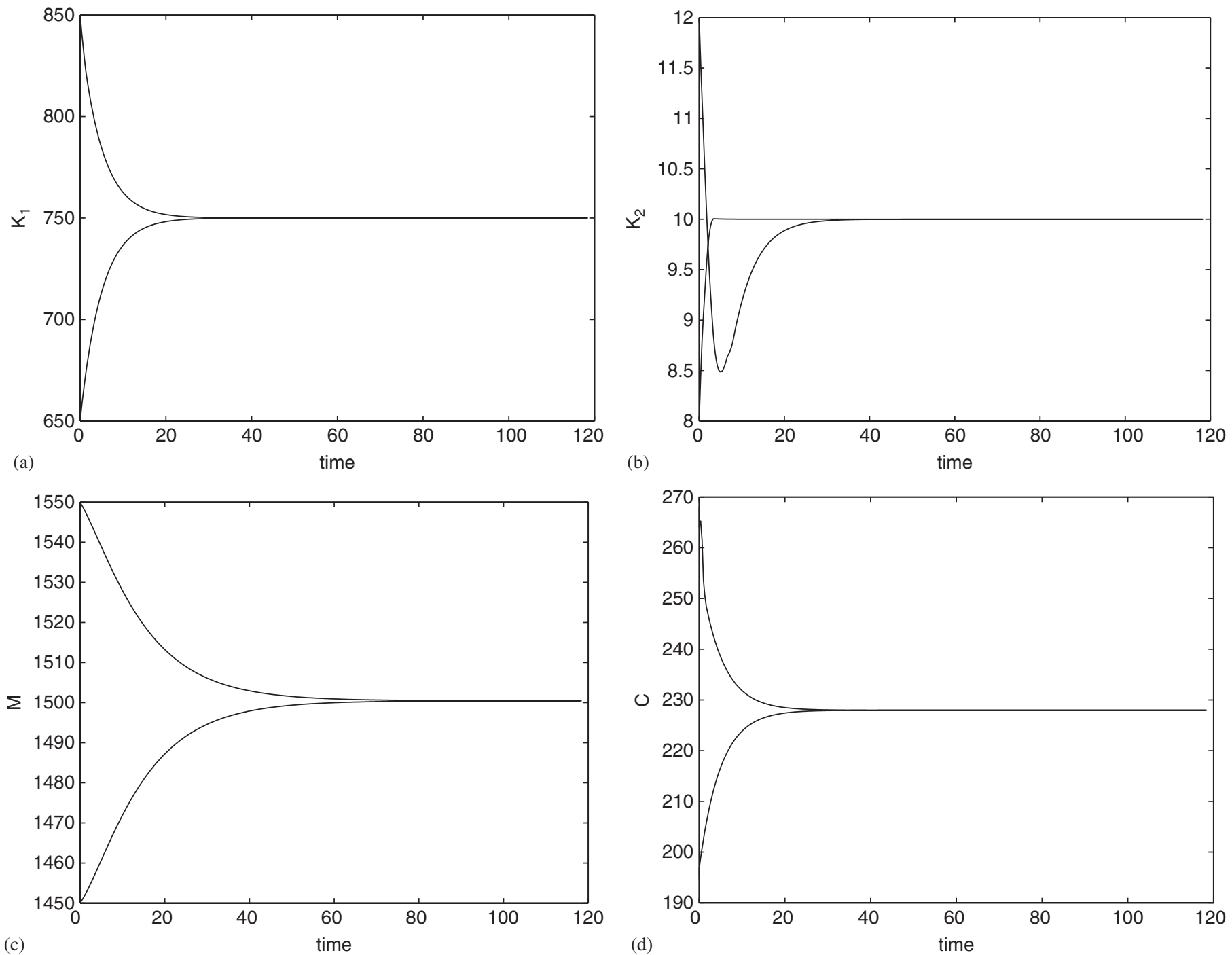

Fig. 1. Two optimal trajectories with different initial values when $\rho=3 \%, \eta_{1}=10^{-3}$ and $\eta_{2}=10^{-6}$. (a) $K_{1}$, (b) $K_{2}$, (c) $M$ and (d) $C$.

Table 7

Turnpike values for $\zeta=0$

\begin{tabular}{llllllll}
\hline$\rho$ & $\eta_{1}$ & $\eta_{2}$ & $K_{1}$ & $K_{2}$ & $M$ & $v$ & $C$ \\
\hline 0.03 & $10^{-3}$ & $1 \times 10^{-6}$ & 750 & 10.0 & 1501 & 0.05 & 228 \\
0.03 & $10^{-3}$ & $5 \times 10^{-6}$ & 700 & 12.5 & 1438 & 0.10 & 231 \\
0.03 & $10^{-3}$ & $1 \times 10^{-5}$ & 650 & 15.0 & 1376 & 0.15 & 234 \\
\hline
\end{tabular}

\section{Acknowledgements}

This research has been supported by the ATLANTIS EU-project and by the Swiss NSF "NCCR-Climate" research program.

\section{References}

Arrow, K. J., \& Kurz, M. (1970). Public investment, rate of return and optimal fiscal policy. Baltimore, MD: Johns Hopkins Press.

Boukas, K., Haurie, A., \& Michel, P. (1990). An optimal control problem with a random stopping time. Journal of Optimization Theory and Application, 64(3), 471-480.
Carlson, D., Haurie, A., \& Leizarowitz, A. (1994). Infinite horizon optimal control: Deterministic and stochastic systems. Berlin: Springer.

Cass, D. (1965). Optimum growth in an aggregative model of capital accumulation. Review of Economic Studies, 32, 233-240.

Cass, D., \& Shell, K. (1976). The structure and stability of competitive dynamical systems. Journal of Economic Theory, 12, 30-70.

Davis, M. H. A. (1984). Piecewise deterministic Markov processes: A general class of non-diffusion stochastic models. Journal of the Roy Statistical Society, 46, 353-388.

Fleming, W. H., \& Rishel, R. W. (1975). Deterministic and stochastic control. Berlin: Springer.

Fleming, W. H., Sethi, S. P., \& Soner, H. M. (1987). An optimal stochastic production planning problem with random fluctuating demand. SIAM Journal of Control and Optimization, 25, 1494-1502.

Fourer, R., Gay, D. M., \& Kernighan, B. W. (1993). AMPL. London: The Scientific Press.

Haurie, A. (2003). Integrated assessment modeling for global climate change: An infinite horizon viewpoint. Environmental Modeling and Assessment, $8(3), 117-132$.

Haurie, A. (2005). A Multigenerational game model to analyze sustainable development. Annals of Operations Research, 137, 369-386.

Haurie, A. (2006). A Stochastic multi-generation game with application to global climate change economic impact assessment. In A. Haurie et al. (eds.). Advances in dynamic games. (vol. 8). Annals of the International Society of Dynamic Games, Basel: Birkhauser. 
Haurie, A., \& Van Delft, Ch. (1995). Turnpikes in flow control models for unreliable manufacturing systems. European Journal of Operational Research, 82, 359-372.

Haurie, A., \& Moresino, F. (to appear). Equilibria in stochastic game models of intergenerational equity. International Game Theory Review.

Kushner, H. J., \& Dupuis, P. G. (1992). Numerical methods for stochastic control problems in continuous time. New York: Springer.

Nordhaus, W. D. (1994). Managing the global commons: The economics of climate change. Cambridge, MA: MIT Press.

Petschel-Held, T., Schellnhuber, H.-J., Bruckner, T., Toth, F. L., \& Hasselmann, K. (1999). The tolerable windows approach: Theoretical and methodological foundations. Climatic Change, 41, 303-331.

Puterman, M. (1994). Markov decision processes. New York: Wiley Interscience.

Ramsey, F. (1928). A mathematic theory of saving. Economic Journal, 38, $543-549$.

Sethi, S. P. (1997). Optimal consumption and investment with bankruptcy. Norwell, MA: Kluwer Academic Publishers.

Tóth, F., Cramer, W., \& Hizsnyik, E. (2000). Climate impact response functions: An introduction. Climatic Change, 46, 225-246.

Vermes, D. (1985). Optimal control of piecewise deterministic Markov process. Stochastics, 14, 165-208.

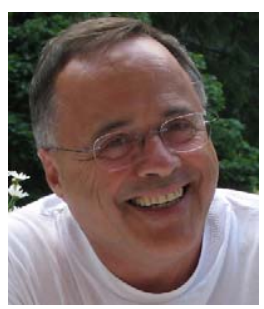

Alain Haurie was born in Algiers, Algeria, on August 26, 1940. He received the Licence es Science degree in mathematics from the University of Algiers, Algeria, in 1961, the Doctorate degree in applied mathematics from the University of Paris VII, Paris, France, in 1970, and the Doctorat es Sciences (Doctorat d'état) degree also from the University of Paris VII in 1976.

Since 1963, he has been a Professor at l'Ecole des Hautes Etudes Commerciales de Montréal, Montréal, P.Q, Canada. In 1976 and 1977, he was on leave of absence at INSEA, Rabat, Morocco.
From 1970 to 1973 and from 1986 to 1988 , he held a part-time teaching and research position in the Department of Mathematics of l'Ecole Polytechnique de Montréal where he was responsible for a graduate course on optimal control theory. In 1979, he held a similar position in the Department of Operations Research, University of Montréal.

From 1980 to 1988, he has been Director of GERAD (Groupe d'Etudes et de Recherche en Analyse de Décisions).

Since 1988, he has been Professor of Operations Research in the Economics and Social Science Faculty of the University of Geneva, Geneva, Switzerland. His current research interests include application of systems analysis and optimization methods to environmental management, application of stochastic control theory to societal problems, application of optimal control theory to economic planning, modeling of manufacturing systems, and dynamic game theory.

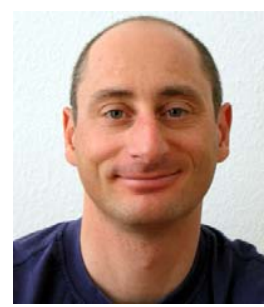

Francesco Moresino holds a degree in physics from ETH Zurich and a Ph.D. in economics from the University of Geneva. He has been a research associate at the Judge Institute of Management Studies, University of Cambridge. He also worked in the risk management solutions team at Reuters and in the department of finance at the private bank EFG. He is currently a research associate at EPFL and works on global environmental management models.

His research interests are in the fields of stochastic optimization and stochastic game theory, with applications to sustainable development, manufacturing and finance. 